\title{
An axiomatic characterization of the Owen-Shapley spatial
}

Citation for published version (APA):

Peters, H. J. M., \& Zarzuelo, J. M. (2015). An axiomatic characterization of the Owen-Shapley spatial. Maastricht University, Graduate School of Business and Economics. GSBE Research Memoranda No. 015 https://doi.org/10.26481/umagsb.2015015

Document status and date:

Published: 01/01/2015

DOI:

10.26481/umagsb.2015015

Document Version:

Publisher's PDF, also known as Version of record

\section{Please check the document version of this publication:}

- A submitted manuscript is the version of the article upon submission and before peer-review. There can be important differences between the submitted version and the official published version of record.

People interested in the research are advised to contact the author for the final version of the publication, or visit the DOI to the publisher's website.

- The final author version and the galley proof are versions of the publication after peer review.

- The final published version features the final layout of the paper including the volume, issue and page numbers.

Link to publication

\footnotetext{
General rights rights.

- You may freely distribute the URL identifying the publication in the public portal. please follow below link for the End User Agreement:

www.umlib.nl/taverne-license

Take down policy

If you believe that this document breaches copyright please contact us at:

repository@maastrichtuniversity.nl

providing details and we will investigate your claim.
}

Copyright and moral rights for the publications made accessible in the public portal are retained by the authors and/or other copyright owners and it is a condition of accessing publications that users recognise and abide by the legal requirements associated with these

- Users may download and print one copy of any publication from the public portal for the purpose of private study or research.

- You may not further distribute the material or use it for any profit-making activity or commercial gain

If the publication is distributed under the terms of Article $25 \mathrm{fa}$ of the Dutch Copyright Act, indicated by the "Taverne" license above, 


\section{Maastricht University}

Hans Peters, José M. Zarzuelo

An axiomatic

characterization of the

Owen-Shapley spatial

power index

$\mathrm{RM} / 15 / 015$

\section{GSBE}

Maastricht University School of Business and Economics

Graduate School of Business and Economics

P.O Box 616

NL- 6200 MD Maastricht

The Netherlands 


\title{
An axiomatic characterization of the Owen-Shapley spatial power index
}

\author{
Hans Peters* José M. Zarzuelo ${ }^{\dagger}$
}

This version, April 2015

\begin{abstract}
We present an axiomatic characterization of the Owen-Shapley spatial power index for the case where issues are elements of two-dimensional space. This characterization employs a version of the transfer condition, which enables us to unravel a spatial game into spatial games connected to unanimity games. The other axioms are spatial versions of anonymity and dummy, and two conditions concerned particularly with the spatial positions of the players. We show that these axioms are logically independent.
\end{abstract}

Journal of Economic Literature Classification Nos.: C71, D72

Keywords: simple game, constellation, spatial game, Owen-Shapley spatial power index

\section{Introduction}

Voting power in political bodies can be represented by simple games, which identify the winning and losing coalitions: a winning coalition can enforce laws, amendments, etc. In order to measure the power of an individual voter or political party one can use a power index, like for instance the Shapley-Shubik index, which is the Shapley value applied to the transferable utility game that assigns one to winning and zero to losing coalitions. As is well known, the Shapley-Shubik index distributes a total of one among the players by assigning to each player the fraction of the number of player permutations in which that player is pivotal. This way of measuring individual power, however, neither takes the possible issues at stake into account nor the individual positions of the players concerning these issues. In order to remedy this defect, Owen and

\footnotetext{
*Department of Quantitative Economics, Maastricht University, Maastricht, The Netherlands. Email: h.peters@maastrichtuniversity.nl.

${ }^{\dagger}$ Department of Applied Economics IV. The University of the Basque Country, Bilbao, Spain. Email: josemanuel.zarzuelo@ehu.es.
} 
Shapley (1989), following up on earlier papers of Owen (1971) and Shapley (1977), assume that each player occupies a position in $\mathbb{R}^{m}$ : one can think of there being $m$ possible criteria, and a point $x \in \mathbb{R}^{m}$ represents a position with respect to these criteria. An issue is then a function $f: \mathbb{R}^{m} \rightarrow \mathbb{R}$, with the interpretation that if $f(x) \leq f(y)$ then a player with position $x$ is more in favor of issue $f$, and thus more likely to support $f$, than a player with position $y$. Owen and Shapley restrict attention to linear homogenous issues; i.e., an issue can be represented by a vector $r \in \mathbb{R}^{m}$ with length one, so that a player with position $x$ is more in favor of $r$ than a player with position $y$ if $r \cdot x \leq r \cdot y$ (dotproducts). Given a simple game and a constellation of player positions, for each issue $r$ a pivotal player exists, by building a coalition starting with the player whose position has the smallest dot-product with $r$. Then the Owen-Shapley spatial power index assigns to each player the fraction of issues at which this player is pivotal. In other words, compared to the Shapley-Shubik index, the player permutations are weighted by considering the positions of the players.

Although the model underlying the Owen-Shapley spatial power index may seem somewhat abstract, the concept derives important support from the fact that, at least if $m=2$ and the simple game is proper and strong (meaning, for each coalition, that either that coalition is winning and its complement losing, or the other way around), the strong point and the center of power coincide. The latter point is the convex combination of the player positions using the Owen-Shapley spatial power index values as weights; and the strong point is the Copeland winner, i.e., the position that is least vulnerable to opposition by (winning) coalitions in terms of Euclidian distance. See Owen and Shapley (1989) for details and a proof of this result.

Also in this paper we assume $m=2$. A spatial game is a combination of a simple game and a constellation of player positions. A spatial power index assigns to each player in a spatial game a nonnegative number, where these numbers sum up to one. We show that the Owen-Shapley spatial power index is uniquely characterized by five axioms: a version of the well known transfer condition similar to the one in Einy and Haimanko (2011); anonymity and dummie axioms adapted to the spatial context; and two axioms dealing exclusively with the spatial positions of the players. We also show that these axioms are logically independent. As far as we are aware, this is the first characterization of the Owen-Shapley spatial power index.

Further related literature. Without claiming completeness, we mention a few relevant related papers. Recently, Martin et al. (2014) have extended the OwenShapley spatial power index to general (sub)sets of issues and player positions. They also clarify the difference and overlap between the original concepts of Owen (1971) and Shapley (1977). Shenoy (1982) provides a spatial version of another well-known power index, the Banzhaf index. Passarelli and Bar (2007) propose a power index which is more directly based on the Euclidian distance of player positions to issues, where the latter are points in the same space as the player positions. They also discuss application to the EU. Benatti and Marzetti (2013) take a generalized approach to power indexes, comprising the Shapley- 
Shubik and Owen-Shapley power indexes, and also apply this to EU's council of ministers. Alonse-Meijide et al. (2011) develop an alternative spatial power index based on lengths of paths connecting player positions and induced by player permutations, and consider an application to the Catalan Parliament.

Also the more specific political science literature provides many interesting references to this topic. We mention in particular Grofman et al. (1987) and Straffin jr (1994) for overviews and applications.

Organization of the paper. Preliminaries are collected in Section 2. Section 3 provides the axioms, characterization, and logical independence, and Section 4 concludes.

\section{Preliminaries}

We introduce the relevant notations and concepts in different subsections.

\subsection{Notations in $\mathbb{R}^{2}$}

For $x, y \in \mathbb{R}^{2}$ with $x \neq y$ we denote by $[x, y, \rightarrow)$ the half-line starting at $x$ and crossing through $y$. The line segment with endpoints $x$ and $y$ is denoted by $[x, y]$; the perpendicular bisector of $[x, y]$ is the line through $\frac{1}{2} x+\frac{1}{2} y$ perpendicular to the line through $x$ and $y$. For a point $x$ and a line $\ell$ in $\mathbb{R}^{2}$ such that $x \notin \ell$ we denote by $x^{\ell}$ the reflection of $x$ with respect to $\ell$, i.e., $\ell$ is the perpendicular bisector of $\left[x, x^{\ell}\right]$. For a point $x \in \ell$ we define $x^{\ell}=x$. By $\bar{x}^{\ell}$ we denote the projection of a point $x$ on a line $\ell$, i.e., $\bar{x}^{\ell}=\frac{1}{2} x+\frac{1}{2} x^{\ell}$. For $x \in \mathbb{R}^{2},\|x\|$ denotes the (Euclidean) length of $x$. For $x=\left(x_{1}, x_{2}\right), y=\left(y_{1}, y_{2}\right) \in \mathbb{R}^{2}, x \cdot y$ denotes the inner product of $x$ and $y$, i.e., $x \cdot y=x_{1} y_{1}+x_{2} y_{2}$. For a subset $X \subseteq \mathbb{R}^{2}$, co $(X)$ denotes the convex hull of $X$.

\subsection{Simple games}

A simple game is a pair $(N, v)$, where $N$ is a nonempty finite subset of $\mathbb{N}$ and $v$ is a function $2^{N} \rightarrow\{0,1\}$ satisfying (a) $v(\emptyset)=0$ and $v(N)=1$; and (b) $v(S) \leq v(T)$ for all $S, T \in 2^{N}$ with $S \subseteq T{ }^{1}$ Elements of $N$ are called players and subsets of $N$ coalitions. A coalition $S$ is winning if $v(S)=1$, otherwise it is losing. A minimal winning coalition is a winning coalition of which each nontrivial subcoalition is losing. We denote by $\mathcal{V}^{N}$ the set of all simple games with player set $N$.

Player $i$ is pivotal in $S$ if $v(S)=1$ and $v(S \backslash\{i\})=0$.

For a simple game $(N, v)$ with at least two players and a player $i \in N$ we define the game $\left(N \backslash\{i\}, v_{-i}\right)$ as follows: $v_{-i}(\emptyset)=0$ and $v_{-i}(S)=v(S \cup\{i\})$ for every $\emptyset \neq S \subseteq N \backslash\{i\}$. The game $\left(N \backslash\{i\}, v_{-i}\right)$ can be interpreted as player $i$ withdrawing from $(N, v)$ but leaving his 'consent' behind; in particular,

\footnotetext{
${ }^{1}$ Thus, in this paper simple games are monotonic by definition.
} 
a winning coalition in $(N, v)$ remains winning in $\left(N \backslash\{i\}, v_{-i}\right)$, i.e., also without player $i$. Note that, indeed, $\left(N \backslash\{i\}, v_{-i}\right) \in \mathcal{V}^{N \backslash\{i\}}{ }^{2}$

\subsection{Constellations}

A constellation for player set $N$ is a vector $p=\left(p_{i}\right)_{i \in N} \in\left(\mathbb{R}^{2}\right)^{N}$ such that $p_{i} \neq p_{j}$ for all $i, j \in N$ with $i \neq j$. We denote by $\mathcal{P}^{N}$ the set of all constellations for player set $N$. For $p \in \mathcal{P}^{N}$ and $i \in N, p_{-i} \in \mathcal{P}^{N \backslash\{i\}}$ is defined by $\left(p_{-i}\right)_{j}=p_{j}$ for every $j \in N \backslash\{i\}$. For a line $\ell$ in $\mathbb{R}^{2}$ and $p \in \mathcal{P}^{N}$, we denote $p^{\ell}=\left(p_{i}^{\ell}\right)_{i \in N}$. Hence, the constellation $p^{\ell}$ is the reflection of the constellation $p$ with respect to $\ell$.

\subsection{Spatial games}

A spatial game for player set $N$ is a triple $(N, v, p)$ where $(N, v) \in \mathcal{V}^{N}$ and $p \in \mathcal{P}^{N}$.

Player $i$ is a dummy in $(N, v, p)$ if $p_{i} \in \operatorname{co}\left(\left\{p_{j} \mid j \in S \backslash\{i\}\right\}\right)$ for every coalition $S$ in which $i$ is pivotal. Note that, in particular, a player who is pivotal in no coalition in the simple game $(N, v)$, is a dummy. More generally, a dummy $i$, even if he is pivotal in some coalition $S$, does not occupy an 'extreme' position in that coalition and therefore will (almost) never exploit this pivotalness: whatever the issue, player $i$ will not be the last player of coalition $S$ to consent. This interpretation is clearly consistent with the idea underlying the Owen-Shapley spatial power index, but it is also consistent with alternative approaches as mentioned in the introduction. For instance, if issues are identified with points in $\mathbb{R}^{2}$ and we take the Euclidian distance as a measure of being close to an issue, then in the situation above player $i$ will never have maximal distance to an issue within the coalition $S$, and thus will never be the last player in $S$ to consent.

\subsection{Power indices}

A spatial power index is a function $\varphi$ which maps each spatial game $(N, v, p)$ to a vector $\varphi(N, v, p) \in \mathbb{R}^{N}$, such that $\varphi_{i}(N, v, p) \geq 0$ for all $i \in N$ and $\sum_{i \in N} \varphi_{i}(N, v, p)=1$. Note, in particular, that a spatial power index is, thus, defined for every (finite) player set $N \subseteq \mathbb{N}$.

Central in this paper is the Owen-Shapley spatial power index (Owen and Shapley, 1989), which we define next.

Let $(N, v, p)$ be a spatial game and let $U=\left\{r \in \mathbb{R}^{2} \mid\|r\|=1\right\}$ denote the circle with radius 1 . We say that player $i \in N$ is pivotal at $r \in U$ if $i$ is pivotal in $S=\left\{j \in N \mid r \cdot p_{j} \leq r \cdot p_{i}\right\}$. Then at each $r \in U$, except at at most finitely many points, there is a unique pivotal player. Let $\rho_{i}$ be the total length of the $\operatorname{arc}(\mathrm{s})$ of $U$ where player $i$ is pivotal, divided by $2 \pi$ (the total length of $U)$; so $\sum_{i \in N} \rho_{i}=1$. Alternatively, $\rho_{i}$ is the probability that player $i$ is pivotal

\footnotetext{
${ }^{2}$ Also note that, even if $(N, v)$ is proper - the complement of each winning coalition is losing - the game $\left(N \backslash\{i\}, v_{-i}\right)$ does not have to be proper.
} 
if $r$ is chosen from the uniform distribution over $U$. The Owen-Shapley spatial power index $\Phi$ now assigns these probabilities to the spatial game $(N, v, p)$ : $\Phi(N, v, p)=\left(\rho_{i}\right)_{i \in N}$.

From a geometric point of view - which we will use often in the sequel player $i$ is pivotal at $r$ if the following holds. Consider a(ny) line $\ell$ in $\mathbb{R}^{2}$ parallel to $r$, and the projections $\bar{p}_{j}^{\ell}$ of the points $p_{j}, j \in N$, on $\ell$. Say that player $j$ precedes player $i$ on $\ell$ if the projection $\bar{p}_{j}^{\ell}$ (weakly) precedes $\bar{p}_{i}^{\ell}$ when going along $\ell$ in the direction of $r$. If player $i$ is pivotal in the set of preceding players (which includes himself), then $i$ is pivotal at $r .^{3}$

To further illustrate the Owen-Shapley spatial power index, we compute it for the case in which the game $v$ is a unanimity game.

Example 2.1. We compute $\Phi$ for the spatial game $\left(N, u_{T}, p\right)$ where $\emptyset \neq T \subseteq N$ and $u_{T}$ is the unanimity game on $T$, i.e., $u_{T}(S)=1$ if $T \subseteq S$ and $u_{T}(S)=0$ otherwise. The players outside $T$ are, trivially, dummies and, in particular, cannot be pivotal at any $r \in U$. Hence, $\Phi_{i}\left(N, u_{T}, p\right)=0$ for all $i \in N \backslash T$ and moreover the points $p_{i}$ for those players play no role in determining $\Phi_{j}\left(N, u_{T}, p\right)$ for the players $j$ in $T$. If $p_{j} \in \operatorname{co}\left(\left\{p_{i} \mid i \in T \backslash\{j\}\right\}\right)$ for some $j \in T$, then again $j$ is a dummy in $\left(N, u_{T}, p\right)$, and for any line $\ell$ the projection $\bar{p}_{j}^{\ell}$ is located between projections of other players in $T$, so that $j$ can be pivotal at at most finitely many $r$; hence $\Phi_{j}\left(N, u_{T}, p\right)=0$. Let $T^{\prime}$ consist of the remaining (non-dummy) players in $T$, and let $P=\operatorname{co}\left(\left\{p_{i} \mid i \in T^{\prime}\right\}\right)$. Thus, $p_{i}$ is an extreme point of $P$ for every $i \in T^{\prime}$. Without loss of generality suppose that $P=\left\{p_{1}, \ldots, p_{k}\right\}$ and that the point $p_{i}$ is adjacent to $p_{i-1}$ and $p_{i+1}$ for each $i \in T^{\prime}$, where $p_{0}=p_{k}$ and $p_{k+1}=p_{1}$. For each $i \in T^{\prime}$ let $\alpha_{i}$ denote the angle (in radians) at $p_{i}$ in $P$.

Let $i \in T^{\prime}$. We compute $\Phi_{i}\left(N, u_{T}, p\right)$. Clearly, if $k=1$ then $\Phi_{i}\left(N, u_{T}, p\right)=$ 1. Now assume that $k \geq 2$. Let $\ell$ be the line through $p_{i-1}$ and $p_{i}$ and let $m$ be the line through $p_{i+1}$ and $p_{i}$. (See Figure 1.) Let $\ell^{\perp}$ be the line through $p_{i}$ perpendicular to $\ell$ and let $m^{\perp}$ be the line through $p_{i}$ perpendicular to $m{ }^{4}$ Consider a vector $r$ of length one starting at $p_{i}$. Then the projections of the points $p_{j}$ for $j \in T^{\prime}$ precede the projection of $p_{i}$ on the line through $p_{i}$ in the direction of $r$ if and only if $r$ is in between $\ell^{\perp}$ and $m^{\perp}$ and pointing outward from $P$. It is easy to see that the (outward) angle between $\ell^{\perp}$ and $m^{\perp}$ is equal to $\pi-\alpha_{i}$, so that $\Phi_{i}\left(N, u_{T}, p\right)=\left(\pi-\alpha_{i}\right) / 2 \pi$. Since the sum of the angles at the vertices of $P$ is equal to $(k-2) \pi$ we have, indeed,

$$
\sum_{i \in T^{\prime}} \Phi_{i}\left(N, u_{T}, p\right)=\frac{1}{2} k-\frac{(k-2) \pi}{2 \pi}=1 .
$$

\footnotetext{
${ }^{3}$ Verification of this claim follows from elementary geometry.

${ }^{4}$ If $k=2$ then $\ell$ and $m$ as well as $\ell^{\perp}$ and $m^{\perp}$ coincide.
} 


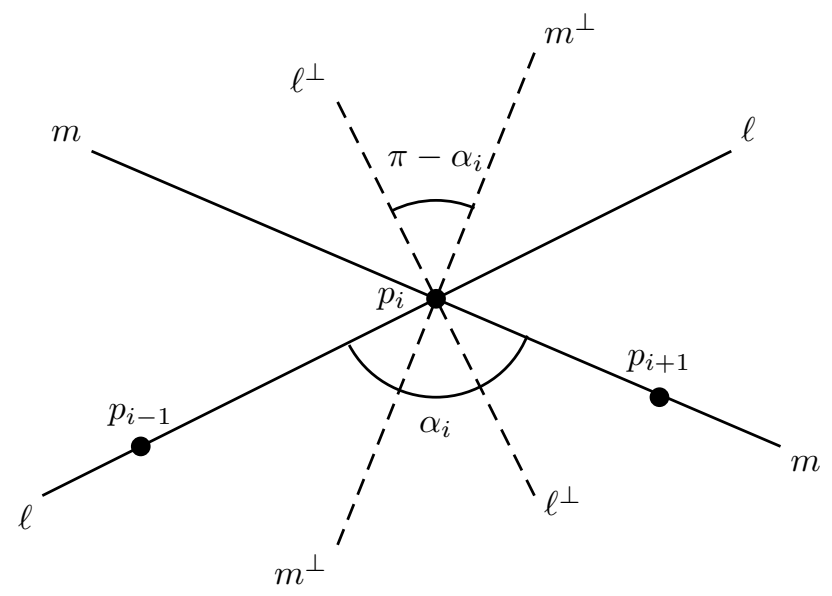

Figure 1: Illustrating Example 2.1

\section{An axiomatic characterization of the Owen- Shapley spatial power index}

We formulate the axioms and state and prove the characterization result. Finally, we show that the axioms are independent.

\subsection{The axioms}

Throughout, let $\varphi$ be a power index. The first axiom is equivalent to the wellknown transfer axiom of Dubey (1975), as remarked in Dubey et al (2005). Here we use a different name which reflects its content in a more direct manner.

Equal Power Change (EPC) For all player sets $N$, all $p \in \mathcal{P}^{N}$, and all $v, v^{\prime}, w, w^{\prime} \in \mathcal{V}^{N}$, if $v-v^{\prime}=w-w^{\prime} \geq 0$, then $\varphi(N, v, p)-\varphi\left(N, v^{\prime}, p\right)=$ $\varphi(N, w, p)-\varphi\left(N, w^{\prime}, p\right)$.

EPC says that, for each constellation, if the same winning coalitions are added when going from $v^{\prime}$ to $v$ as when going from $w^{\prime}$ to $w$, then the change in power for every player when going from $v^{\prime}$ to $v$ should be equal to the change in power when going from $w^{\prime}$ to $w$. As in Einy and Haimanko (2011), the condition will imply that, for each fixed constellation, the power index for arbitrary simple games is completely determined by its value on unanimity games as in Example 2.1.

The second axiom requires that dummies can be left out - while leaving behind their consent - without any effect on the power of the remaining players.

Dummy Property (DP) For every spatial game $(N, v, p)$ and every dummy $i$ in $(N, v, p), \varphi_{j}(N, v, p)=\varphi_{j}\left(N \backslash\{i\}, v_{-i}, p_{-i}\right)$ for every $j \in N \backslash\{i\}$. 
The Dummy Property is a natural consequence of the definition and interpretation of a dummy in Sect. 2.4. It is similar in spirit to the Null-Player-Out property of Derks and Haller (1999), in that it requires that dummies not only have zero power (which is a direct consequence of DP) but can be left out, in the way specified, without changing the power of the remaining players. In Section 3.3 we will demonstrate that it is not sufficient to require only that dummies have zero power.

The third axiom is a standard anonymity requirement, expressing that it should not matter how the players in a spatial game are called. Let $N \subseteq \mathbb{N}$ be finite and let $\sigma: N \rightarrow \mathbb{N}$ be an injective function. For a spatial game $(N, v, p)$ define the spatial game $(\sigma(N), \sigma v, \sigma p)$ by $\sigma v(\sigma(S))=v(S)$ for all $S \subseteq N$ and $(\sigma p)_{\sigma(i)}=p_{i}$ for all $i \in N$.

Anonymity (AN) For every spatial game $(N, v, p)$ and every injective function $\sigma: N \rightarrow \mathbb{N}, \varphi_{\sigma(i)}(\sigma(N), \sigma v, \sigma p)=\varphi_{i}(N, v, p)$ for all $i \in N$.

The last two axioms concern constellations. The first implies that it does not matter where in $\mathbb{R}^{2}$ the constellation is located. It is a natural consequence of the assumption that for a power index all potential issues are taken into consideration and are regarded equally likely, so that only the relative positions of the players matter. Without this condition, other distributions of issues than the uniform one become possible. (Cf. Section 3.3.)

Reflection Invariance (RI) For every spatial game $(N, v, p)$ and every line $\ell$ in $\mathbb{R}^{2}, \varphi(N, v, p)=\varphi\left(N, v, p^{\ell}\right)$.

The final axiom reflects the consideration that what matters in a constellation are the relative positions of the players as expressed by the lines connecting them. The axiom states that the power of a player $i$ does not change if the position $p_{j}$ of another player $j$ moves along the line through $p_{i}$ and $p_{j}$ without passing $p_{i}$. Note that this implies that the order of precedence of the projections of $p_{i}$ and $p_{j}$ on any line does not change. Thus, while all five axioms will be crucial for obtaining the Owen-Shapley spatial power index - see Sect. 3.3 - the final axiom perhaps most closely reflects the idea underlying this index.

Positional Invariance (PI) For all player sets $N, i \in N$, and $p, p^{\prime} \in \mathcal{P}^{N}$, if $p_{i}^{\prime}=p_{i}$ and $p_{j}^{\prime} \in\left[p_{i}, p_{j}, \rightarrow\right)$ for all $j \in N \backslash\{i\}$, then $\varphi_{i}(N, v, p)=\varphi_{i}\left(N, v, p^{\prime}\right)$ for all $(N, v) \in \mathcal{V}^{N}$.

Positional Invariance expresses that issues are identified with lines: if position $p_{j}$ moves along the line through $p_{i}$ and $p_{j}$ in the direction of $p_{i}$ or away from $p_{i}$, then the relative positions of $i$ and $j$ with respect to the issue represented by that line do not change.

\subsection{The characterization}

The main result of this paper is the following. 
Theorem 3.1. The Owen-Shapley spatial power index $\Phi$ is the unique spatial power index satisfying $E P C, D P, A N, R I$, and $P I$.

We first show that $\Phi$ satisfies the five axioms in the theorem.

Lemma 3.2. $\Phi$ satisfies EPC.

Proof. To show that $\Phi$ satisfies EPC, let $p \in \mathcal{P}^{N}$ and let $v, v^{\prime}, w, w^{\prime}$ be simple games with player set $N$, satisfying $v-v^{\prime}=w-w^{\prime} \geq 0$. It is sufficient to consider the case where $v$ arises from $v^{\prime}$ and $w$ from $w^{\prime}$ by adding the minimal winning coalition $S$. First consider a player $i \in S$. Then $v(T \cup\{i\})-v(T) \geq$ $v^{\prime}(T \cup\{i\})-v^{\prime}(T)$ and $w(T \cup\{i\})-w(T) \geq w^{\prime}(T \cup\{i\})-w^{\prime}(T)$ for all $T \subseteq N \backslash\{i\}$. This implies that, according to $\Phi$, player $i$ can never lose power when going from $v^{\prime}$ to $v$ and from $w^{\prime}$ to $w$. Player $i$ gains, when going from $v^{\prime}$ to $v$, at an $r \in U$ where he is not pivotal according to $v^{\prime}$ but pivotal according to $v$. Suppose the induced ordering at such an $r$ is $i_{1}, \ldots, i_{n}$ (where $n=|N|$ ), player $i_{t}$ is pivotal according to $v^{\prime}, S \subseteq\left\{i_{1}, \ldots, i_{t-1}\right\}$, and $i$ is the last player of $S$ in this ordering, say $i=i_{s}$ for some $s \leq t-1$. Then $\left\{i_{1}, \ldots, i_{s}\right\}$ is losing in $v^{\prime}$ and winning in $v$, and therefore losing in $w^{\prime}$ and winning in $w$. Also, $\left\{i_{1}, \ldots, i_{s-1}\right\}$ is losing in $v^{\prime}, v, w^{\prime}$, and therefore also in $w$. Thus, player $i$ is pivotal according to $v$ and according to $w$ but not according to $v^{\prime}$ and $w^{\prime}$. Hence, the gain in power for player 1 at this particular ordering when going from $v^{\prime}$ to $v$ and when going from $w^{\prime}$ to $w$ is equal to the share of the vectors $r \in U$ inducing this ordering, which depends exclusively on the constellation $p$. We conclude that the gain of each player $i \in S$ when going from $v^{\prime}$ to $v$ and when going from $w^{\prime}$ to $w$ is the same.

Next, let $i \notin S$. Now player $i$ can only lose when going from $v^{\prime}$ to $v$ and from $w^{\prime}$ to $w$. Player $i$ loses, when going from $v^{\prime}$ to $v$, at an $r \in U$ where he is pivotal according to $v^{\prime}$ but not pivotal according to $v$. Suppose the induced ordering at such an $r$ is $i_{1}, \ldots, i_{n}$ (where $n=|N|$ ), player $i=i_{t}$ is pivotal according to $v^{\prime}$, and $S \subseteq\left\{i_{1}, \ldots, i_{t-1}\right\}$, so that player $i$ is no longer pivotal according to $v$. Since $S$ is also winning in $w$, player $i$ is also not pivotal according to $w$. Since $\left\{i_{1}, \ldots, i_{t-1}\right\}$ is losing in $v^{\prime}$ and winning in $v$, it must be losing in $w^{\prime}$; and since $\left\{i_{1}, \ldots, i_{t}\right\}$ is winning in $v^{\prime}, v$, and $w$, it must be winning in $w^{\prime}$. Hence, player $i$ is pivotal according to $w^{\prime}$. Hence, the loss in power for player 1 at this particular ordering when going from $v^{\prime}$ to $v$ and when going from $w^{\prime}$ to $w$ is equal to the share of the vectors $r \in U$ inducing this ordering, which depends exclusively on the constellation $p$. We conclude that the loss of each player $i \in S$ when going from $v^{\prime}$ to $v$ and when going from $w^{\prime}$ to $w$ is the same.

Lemma 3.3. $\Phi$ satisfies $D P, A N, R I$, and $P I$.

Proof. Let $(N, v, p)$ be a spatial game. If player $i$ is a dummy, i.e., $p_{i} \in \operatorname{co}\left(\left\{p_{j} \mid\right.\right.$ $j \in S \backslash\{i\}\}$ ) for every coalition $S$ in which $i$ is pivotal, then the projection of $p_{i}$ on any line $\ell$ is in between the projections of other players in $S$, for every $S$ in which $i$ is pivotal. Hence, player $i$ is pivotal at at most finitely many $r \in U$. Thus, $\Phi_{i}(N, v, p)=0$ and nothing changes for the other players if we leave out 
player $i$ from $(N, v)$ and $p_{i}$ from $p$, i.e., $\varphi_{j}(N, v, p)=\varphi_{j}\left(N \backslash\{i\}, v_{-i}, p_{-i}\right)$ for every $j \in N \backslash\{i\}$. Hence, $\Phi$ satisfies DP.

AN of $\Phi$ is obvious by definition. For RI, suppose that player $i$ in $(N, v, p)$ is pivotal at some $r \in U$. Let $\ell$ be a line in $\mathbb{R}^{2}$, and let $m$ be the line through the origin parallel to $\ell$. Then it is easy to check that player $i$ is pivotal at $r^{m} \in U$ in the spatial game $\left(N, v, p^{\ell}\right)$. Since this holds for every player $i$ and every $r \in U$, RI of $\Phi$ follows.

Finally, let $p^{\prime} \in \mathcal{P}^{N}$ and $i \in N$ such that $p_{i}^{\prime}=p_{i}$ and $p_{j}^{\prime} \in\left[p_{i}, p_{j}, \rightarrow\right)$ for all $j \in N \backslash\{i\}$. Then, for every $r \in U$, player $i$ is pivotal at $r$ in $(N, v, p)$ if and only if he is pivotal at $r$ in $\left(N, v, p^{\prime}\right)$, since the set of preceding players of player $i$ does not change. Therefore, $\varphi_{i}(N, v, p)=\varphi_{i}\left(N, v, p^{\prime}\right)$, and PI of $\Phi$ is proved.

Lemmas 3.2 and 3.3 imply that the Owen-Shapley spatial power index satisfies the five axioms in the theorem. We now show uniqueness.

The next lemma implies that, if $\varphi$ satisfies EPC, then it is completely determined by its value on unanimity games as defined in Example 2.1. The lemma follows from Lemma 2.3 in Einy (1987), see also Einy and Haimanko (2011).

Lemma 3.4. Let $\varphi$ be a spatial power index satisfying EPC and let $(N, v, p)$ be a spatial game. Let $T_{1}, \ldots, T_{k}$ be the minimal winning coalitions in $(N, v)$. Then

$$
\varphi(N, v, p)=\sum_{\emptyset \neq I \subseteq\{1, \ldots, k\}}(-1)^{|I|+1} \varphi\left(N, u_{\cup_{m \in I} T_{m}}, p\right) .
$$

Until further notice, $\varphi$ is a spatial power index satisfying DP, AN, RI, and PI.

Lemma 3.5. Let $\left(N, u_{T}, p\right)$ be a spatial game, where $\emptyset \neq T \subseteq N$. Let $T^{\prime} \subseteq$ $N$ be the set of non-dummies. Then $\varphi_{i}(N, v, p)=0$ for all $i \in N \backslash T^{\prime}$ and $\varphi_{i}\left(N, u_{T}, p\right)=\varphi_{i}\left(T^{\prime}, u_{T^{\prime}}, p^{\prime}\right)$ for all $i \in T^{\prime}$, where $p^{\prime}$ is the restriction of $p$ to $T^{\prime}$.

Proof. Clearly, $T^{\prime} \subseteq T$. Let $j \in N \backslash T^{\prime}$, then $\varphi_{i}\left(N, u_{T}, p\right)=\varphi_{i}(N \backslash$ $\left.\{j\},\left(u_{T}\right)_{-j}, p_{-j}\right)$ for all $i \in N \backslash\{j\}$ by DP. In particular, $\varphi_{j}(N, v, p)=1-$ $\sum_{i \in N \backslash\{j\}} \varphi_{i}\left(N \backslash\{j\},\left(u_{T}\right)_{-j}, p_{-j}\right)=1-1=0$; and $\varphi_{i}\left(N, u_{T}, p\right)=\varphi_{i}(N \backslash$ $\left.\{j\}, u_{T \backslash\{j\}}, p_{-j}\right)$ for all $i \in N \backslash\{j\}$. Also, $T^{\prime}$ is still the set of non-dummies in $\left(N \backslash\{j\}, u_{T \backslash\{j\}}, p_{-j}\right)$. The lemma now follows from repeated application of this argument.

In view of Lemma 3.5 we may restrict attention to spatial games of the form $\left(N, u_{N}, p\right)$ and having no dummies. The next lemma establishes a very useful consequence of $\mathrm{AN}$ and $\mathrm{RI}$ for this kind of spatial game.

Lemma 3.6. Let $\left(N, u_{N}, p\right)$ be a spatial game and suppose that for two distinct players $i$ and $j$ with $\ell$ the perpendicular bisector of $\left[p_{i}, p_{j}\right]$ we have $\left\{p_{k}^{\ell} \mid k \in\right.$ $N\}=\left\{p_{k} \mid k \in N\right\}$. Then $\varphi_{i}\left(N, u_{N}, p\right)=\varphi_{j}\left(N, u_{N}, p\right)$. 


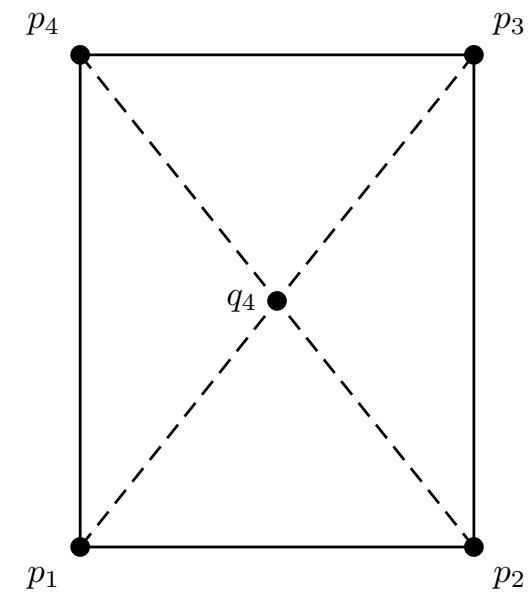

Figure 2: Illustrating the proof of Lemma 3.8

Proof. Consider $\sigma: N \rightarrow N$ defined by $\sigma(k)=k^{\prime}$ if $p_{k^{\prime}}=p_{k}^{\ell}$. By AN, $\varphi_{i}\left(N, u_{N}, p\right)=\varphi_{\sigma(i)}\left(N, \sigma u_{N}, \sigma p\right)=\varphi_{j}\left(N, u_{N}, p^{\ell}\right)$. By RI, $\varphi_{j}\left(N, u_{N}, p^{\ell}\right)=$ $\varphi_{j}\left(N, u_{N}, p\right)$. Hence $\varphi_{i}\left(N, u_{N}, p\right)=\varphi_{j}\left(N, u_{N}, p\right)$.

Lemma 3.7. Let $\left(N, u_{N}, p\right)$ be a spatial game. If $|N|=1$ or $|N|=2$ then $\varphi\left(N, u_{N}, p\right)=\Phi\left(N, u_{N}, p\right)$.

Proof. Observe that in both cases there are no dummies. If $|N|=\{i\}$ then clearly $\varphi_{i}\left(N, u_{N}, p\right)=\Phi_{i}\left(N, u_{N}, p\right)=1$. If $N=\{i, j\}$ with $j \neq i$ then $\varphi_{i}\left(N, u_{N}, p\right)=\varphi_{j}\left(N, u_{N}, p\right)$ by Lemma 3.6. This holds also for $\Phi$, and we obtain $\Phi_{i}\left(N, u_{N}, p\right)=\Phi_{j}\left(N, u_{N}, p\right)=\varphi_{i}\left(N, u_{N}, p\right)=\varphi_{j}\left(N, u_{N}, p\right)=\frac{1}{2}$.

Lemma 3.8. Let $|N|=3, k \in N, p \in \mathcal{P}^{N}$, and let $\operatorname{co}\left(\left\{p_{i} \mid i \in N\right\}\right)$ have a right angle at $p_{k}$. Then $\varphi_{k}\left(N, u_{N}, p\right)=\Phi_{k}\left(N, u_{N}, p\right)=\frac{1}{4}$.

Proof. (Cf. Figure 2.) Without loss of generality let $N=\{1,2,3\}$ with $k=2$ and consider an additional player 4 with $p_{4} \in \mathbb{R}^{2}$ such that $\operatorname{co}\left(\left\{p_{i} \mid i \in N \cup\right.\right.$ $\{4\}\})$ is a rectangle with vertex $p_{4}$ opposite of $p_{2}$. Consider the spatial game $\left(N \cup\{4\}, u_{N \cup\{4\}}, p\right)$. By Lemma 3.6 we obtain $\varphi_{1}\left(N \cup\{4\}, u_{N \cup\{4\}}, p\right)=\varphi_{2}(N \cup$ $\left.\{4\}, u_{N \cup\{4\}}, p\right)$, and similarly $\varphi_{2}\left(N \cup\{4\}, u_{N \cup\{4\}}, p\right)=\varphi_{3}\left(N \cup\{4\}, u_{N \cup\{4\}}, p\right)=$ $\varphi_{4}\left(N \cup\{4\}, u_{N \cup\{4\}}, p\right)$, so that $\varphi_{i}\left(N \cup\{4\}, u_{N \cup\{4\}}, p\right)=\frac{1}{4}$ for every $i=1, \ldots, 4$.

Next consider the spatial game $\left(N \cup\{4\}, u_{N \cup\{4\}}, q\right)$, where $q_{i}=p_{i}$ for $i=1,2,3$ and $q_{4}=\frac{1}{2} p_{1}+\frac{1}{2} p_{3}$. By PI, $\varphi_{2}\left(N \cup\{4\}, u_{N \cup\{4\}}, q\right)=\varphi_{2}(N \cup$ $\left.\{4\}, u_{N \cup\{4\}}, p\right)=\frac{1}{4}$. Since player 4 is a dummy in $\left(N \cup\{4\}, u_{N \cup\{4\}}, q\right)$, we obtain $\varphi_{2}\left(N, u_{N}, p\right)=\varphi_{2}\left(N,\left(u_{N \cup\{4\}}\right)_{-4}, q_{-4}\right)=\varphi_{2}\left(N \cup\{4\}, u_{N \cup\{4\}}, q\right)=\frac{1}{4}$, where the second equality follows from DP. The same argument (or Example 2.1) applies to $\Phi$.

In what follows we use the notation $\measuredangle x y z$ for the size of the (smaller) angle, measured in radians, at $y$ between the line through $x$ and $y$ and the line through $z$ and $y$. The following lemma and its proof are illustrated in Figure 3. 


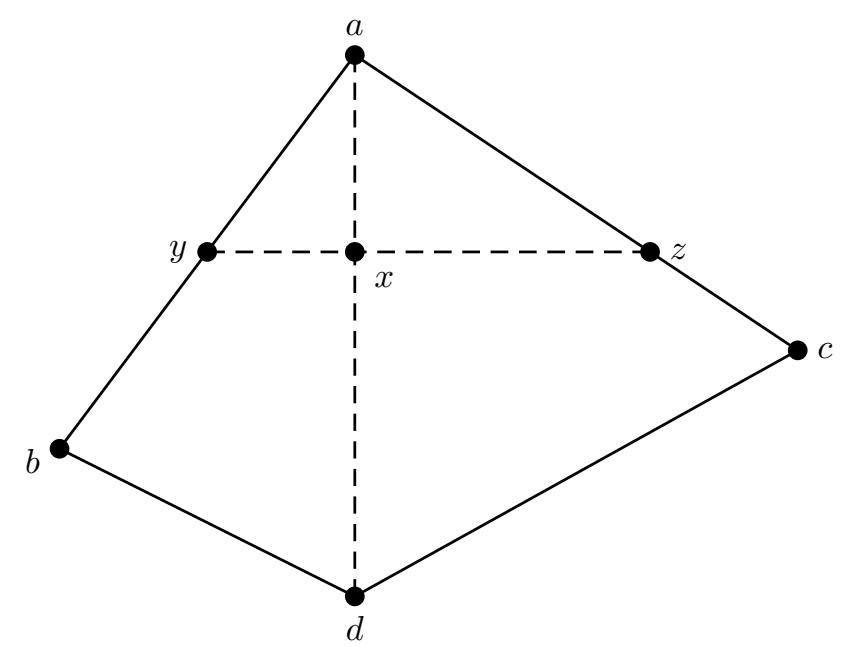

Figure 3: Illustrating Lemma 3.9 and its proof

Lemma 3.9. Let $a, b, c$, and $d$ be four distinct points in $\mathbb{R}^{2}$ such that $b$ and $c$ are on different sides of the line through $a$ and $d$, and $\measuredangle b a d+\measuredangle c a d<\pi$. Let $N=\{i, j, k\},|N|=3$, and let $p, q, s \in \mathcal{P}^{N}$ be defined by $p=\left(p_{i}, p_{j}, p_{k}\right)=$ $(a, b, c), q=\left(q_{i}, q_{j}, q_{k}\right)=(a, b, d)$, and $s=\left(s_{i}, s_{j}, s_{k}\right)=(a, d, c)$, Then

$$
\varphi_{i}\left(N, u_{N}, p\right)=\varphi_{i}\left(N, u_{N}, q\right)+\varphi_{i}\left(N, u_{N}, s\right)-\frac{1}{2} .
$$

If, additionally, $\measuredangle b a d=\measuredangle c a d$, then

$$
\varphi_{i}\left(N, u_{N}, p\right)=2 \varphi_{i}\left(N, u_{N}, q\right)-\frac{1}{2} .
$$

Proof. Take a point $x$ in the open line segment between $a$ and $d$, such that the line through $x$ perpendicular to the line through $a$ and $d$ intersects the open line segment between $a$ and $b$ in a point $y$ and the open line segment between $a$ and $c$ in a point $z$. By PI it is sufficient to prove that

$$
\varphi_{i}\left(N, u_{N}, \widetilde{p}\right)=\varphi_{i}\left(N, u_{N}, \widetilde{q}\right)+\varphi_{i}\left(N, u_{N}, \widetilde{s}\right)-\frac{1}{2},
$$

and

$$
\varphi_{i}\left(N, u_{N}, \widetilde{p}\right)=2 \varphi_{i}\left(N, u_{N}, \widetilde{q}\right)-\frac{1}{2}
$$

if $\measuredangle b a d=\measuredangle c a d$, where $\widetilde{p}=\left(\widetilde{p}_{i}, \widetilde{p}_{j}, \widetilde{p}_{k}\right)=(a, y, z), \widetilde{q}=\left(\widetilde{q}_{i}, \widetilde{q}_{j}, \widetilde{q}_{k}\right)=(a, y, x)$, and $\widetilde{s}=\left(\widetilde{s}_{i}, \widetilde{s}_{j}, \widetilde{s}_{k}\right)=(a, x, z)$. Now

$$
\varphi_{j}\left(N, u_{N}, \widetilde{p}\right)=\varphi_{j}\left(N, u_{N}, \widetilde{q}\right)=\frac{3}{4}-\varphi_{i}\left(N, u_{N}, \widetilde{q}\right),
$$

where the first equality follows from PI and the second from Lemma 3.8. By analogous arguments,

$$
\varphi_{k}\left(N, u_{N}, \widetilde{p}\right)=\varphi_{k}\left(N, u_{N}, \widetilde{s}\right)=\frac{3}{4}-\varphi_{i}\left(N, u_{N}, \widetilde{s}\right) .
$$


Combining (1) and (2) we obtain

$$
\begin{aligned}
\varphi_{i}\left(N, u_{N}, \widetilde{p}\right) & =1-\varphi_{j}\left(N, u_{N}, \widetilde{p}\right)-\varphi_{k}\left(N, u_{N}, \widetilde{p}\right) \\
& =\varphi_{i}\left(N, u_{N}, \widetilde{q}\right)+\varphi_{i}\left(N, u_{N}, \widetilde{s}\right)-\frac{1}{2},
\end{aligned}
$$

which completes the proof of the first statement. Now suppose, additionally, that $\measuredangle b a d=\measuredangle c a d$. Then by Lemma 3.6 we have $\varphi_{j}\left(N, u_{N}, \widetilde{p}\right)=\varphi_{k}\left(N, u_{N}, \widetilde{p}\right)$. By (1) and (2) this implies $\varphi_{i}\left(N, u_{N}, \widetilde{q}\right)=\varphi_{i}\left(N, u_{N}, \widetilde{s}\right)$, and the second statement in the lemma follows.

Lemma 3.10. Let $N=\{i, j, k\},|N|=3$, and let $p \in \mathcal{P}^{N}$ such that $\measuredangle p_{j} p_{i} p_{k}=$ $\frac{m}{2^{h}} \pi$ for some $h, m \in \mathbb{N}$ with $m<2^{h}$. Then $\varphi_{i}\left(N, u_{N}, p\right)=\frac{1}{2}-\frac{m}{2^{h+1}}$.

Proof. First suppose $m=1$. If $h=1$ then $\varphi_{i}\left(N, u_{N}, p\right)=\frac{1}{4}$ by Lemma 3.8. Assume that the statement has been proved for $h$, then for $h+1$ we have by the second statement in Lemma 3.9 that

$$
\frac{1}{2}-\frac{1}{2^{h+1}}=2 \varphi_{i}\left(N, u_{N}, p\right)-\frac{1}{2},
$$

hence $\varphi_{i}\left(N, u_{N}, p\right)=\frac{1}{2}-\frac{1}{2^{h+2}}$. By induction the statement in the lemma follows for $m=1$ and all $h \in \mathbb{N}$. Now suppose the statement in the lemma has been proved for $m$ and arbitrary $h$ such that $m+1<2^{h}$, Then by the first statement in Lemma 3.9 we obtain for $m+1$

$$
\varphi_{i}\left(N, u_{N}, p\right)=\left(\frac{1}{2}-\frac{1}{2^{h+1}}\right)+\left(\frac{1}{2}-\frac{m}{2^{h+1}}\right)-\frac{1}{2}=\frac{1}{2}-\frac{m+1}{2^{h+1}}
$$

and the proof of the lemma is complete by induction.

Let

$$
A=\left\{\frac{m}{2^{h}} \pi \mid h, m \in \mathbb{N}, m<2^{h}\right\} .
$$

With some abuse of notation we will write $\varphi(\alpha):=\varphi_{i}\left(N, u_{N}, p\right)$ for $\left(N, u_{N}, p\right)$ as in Lemma 3.10, where $\alpha=\measuredangle p_{j} p_{i} p_{k} \in A$. Observe that $A$ is a dense subset of the interval $(0, \pi)$ and that $\varphi: A \rightarrow\left(0, \frac{1}{2}\right), \frac{m}{2^{h}} \pi \mapsto \frac{1}{2}-\frac{m}{2^{h+1}}$ is a decreasing continuous function. Note that $\varphi(\alpha)=\frac{\pi-\alpha}{2 \pi}$ for every $\alpha \in A$.

Next, we extend these findings to all of $(0, \pi)$.

Lemma 3.11. Let $a, b, c$, and $d$ be four distinct points in $\mathbb{R}^{2}$ such that $d$ and $b$ are on different sides of the line through $a$ and $c$, and $0<\measuredangle c a b<\measuredangle d a b<\pi$. Let $N=\{i, j, k\},|N|=3$, let $p=\left(p_{i}, p_{j}, p_{k}\right)=(a, b, c)$ and let $q=\left(q_{i}, q_{j}, q_{k}\right)=$ $(a, b, d)$. Then $\varphi_{i}\left(N, u_{N}, p\right) \geq \varphi_{i}\left(N, u_{N}, q\right)$.

Proof. By PI we may assume that the line $m$ through $b$ and $d$ is perpendicular to the line $\ell$ through $a$ and $c$ and that these two lines intersect in $c$. See Figure 4 for an illustration of this and of the later part of the proof. Without loss of generality we assume $i=1, j=2, k=3$. 


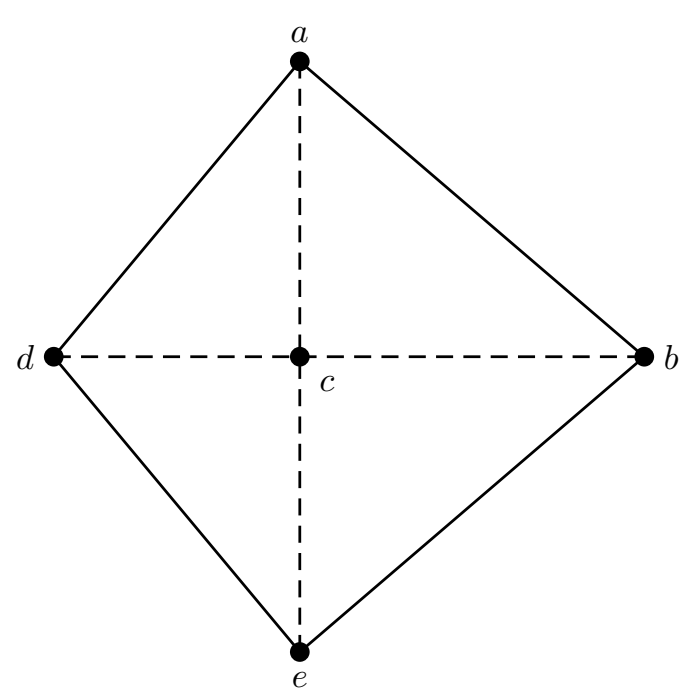

Figure 4: Illustrating the proof of Lemma 3.11

Consider the spatial game $\left(N \cup\{4\}, u_{N \cup\{4\}},(a, b, e, d)\right)$, where $e=a^{m}$. By PI and DP we obtain

$$
\varphi_{2}\left(N \cup\{4\}, u_{N \cup\{4\}},(a, b, e, d)\right)=\varphi_{2}\left(N, u_{N},(a, b, e)\right)
$$

which can be seen by moving the location $d$ of player 4 to $c$ along $m$. Since $\varphi_{1}\left(N, u_{N},(a, b, e)\right)=\varphi_{3}\left(N, u_{N},(a, b, e)\right)$ by Lemma 3.6, we have

$$
\varphi_{2}\left(N, u_{N},(a, b, e)\right)=1-2 \varphi_{1}\left(N, u_{N},(a, b, e)\right) .
$$

Since also $\varphi_{1}\left(N \cup\{4\}, u_{N \cup\{4\}},(a, b, e, d)\right)=\varphi_{3}\left(N \cup\{4\}, u_{N \cup\{4\}},(a, b, e, d)\right)$ by Lemma 3.6, we obtain

$$
\begin{aligned}
\varphi_{2}\left(N \cup\{4\}, u_{N \cup\{4\}},(a, b, e, d)\right)= & 1-2 \varphi_{1}\left(N \cup\{4\}, u_{N \cup\{4\}},(a, b, e, d)\right) \\
& -\varphi_{4}\left(N \cup\{4\}, u_{N \cup\{4\}},(a, b, e, d)\right) .
\end{aligned}
$$

Combining (3), (4), (5) and the assumption that $\varphi_{4}\left(N \cup\{4\}, u_{N \cup\{4\}},(a, b, e, d)\right) \geq$ 0 , we obtain

$$
\varphi_{1}\left(N, u_{N},(a, b, e)\right) \geq \varphi_{1}\left(N \cup\{4\}, u_{N \cup\{4\}},(a, b, e, d)\right) .
$$

By PI, $\varphi_{1}\left(N, u_{N},(a, b, e)\right)=\varphi_{1}\left(N, u_{N},(a, b, c)\right)$ and by PI and DP, $\varphi_{1}(N \cup$ $\left.\{4\}, u_{N \cup\{4\}},(a, b, e, d)\right)=\varphi_{1}\left(\{1,2,4\}, u_{\{1,2,4\}},(a, b, d)\right.$. Together with (6) we have $\varphi_{1}\left(N, u_{N},(a, b, c)\right) \geq \varphi_{1}\left(\{1,2,4\}, u_{\{1,2,4\}},(a, b, d)\right.$, hence by AN, $\varphi_{1}(N$, $\left.u_{N},(a, b, c)\right) \geq \varphi_{1}\left(N, u_{N},(a, b, d)\right.$.

Lemma 3.11 in fact states that in a spatial game $\left(N, u_{N}, p\right)$ with three players and the points $p_{k}$ forming a triangle, the power of player $i$ decreases as the angle at the point $p_{i}$ increases. Combined with Lemma 3.10 and the discussion following it we thus obtain the following result.

Corollary 3.12. Let $N=\{i, j, k\},|N|=3$, and let $p \in \mathcal{P}^{N}$. Then for each $i \in N$,

$$
\Phi_{i}\left(N, u_{N}, p\right)=\varphi_{i}\left(N, u_{N}, p\right)=\frac{\pi-\measuredangle p_{j} p_{i} p_{k}}{2 \pi} .
$$


The case with an arbitrary number of players now follows easily.

Corollary 3.13. Let $\left(N, u_{N}, p\right)$ be a spatial game without dummies and let $i, j, k \in N$ such that $p_{j}$ and $p_{k}$ are adjacent to $p_{i}$. Then

$$
\Phi_{i}\left(N, u_{N}, p\right)=\varphi_{i}\left(N, u_{N}, p\right)=\frac{\pi-\measuredangle p_{j} p_{i} p_{k}}{2 \pi} .
$$

Proof. By PI and DP, $\varphi_{i}\left(N, u_{N}, p\right)=\varphi_{i}\left(\{i, j, k\}, u_{\{i, j, k\}},\left(p_{i}, p_{j}, p_{k}\right)\right)$. The claim now follows from Corollary 3.12.

Proof of Theorem 3.1 By Lemmas 3.2 and 3.3, $\Phi$ satisfies all the axioms in the theorem. The converse follows from Corollary 3.13 and Lemma 3.5, combined with Lemma 3.4.

\subsection{Independence of the axioms}

The following spatial power indices show that the axioms in Theorem 3.1 are logically independent.

(1) Define

$$
\varphi^{1}(N, v, p)=\frac{1}{2^{k}-1} \sum_{\emptyset \neq I \subseteq\{1, \ldots, k\}} \Phi\left(N, u_{\cup_{m \in I} T_{m}}, p\right)
$$

for every spatial game $(N, v, p)$, where $T_{1}, \ldots, T_{k}$ are the minimal winning coalitions in $(N, v)$ (cf. Lemma 3.4). Then $\varphi^{1}$ satisfies all the axioms in Theorem 3.1 except EPC.

(2) Define $\varphi_{i}^{2}(N, v, p)=1 /|N|$ for every spatial game $(N, v, p)$ and every $i \in N$. Then $\varphi^{2}$ satisfies all the axioms in Theorem 3.1 except DP.

(3) For every spatial game $\left(N, u_{T}, p\right)$ where the set of non-dummies is equal to $\{1,2\}$, let $\varphi_{1}^{3}\left(N, u_{T}, p\right)=\frac{1}{4}, \varphi_{2}^{3}\left(N, u_{T}, p\right)=\frac{3}{4}$, and $\varphi_{i}^{3}\left(N, u_{T}, p\right)=0$ for all $i \in N \backslash\{1,2\}$. In all other cases, let $\varphi^{3}\left(N, u_{T}, p\right)=\Phi\left(N, u_{T}, p\right)$. Extend $\varphi^{3}$ to a spatial power index by using the formula in Lemma 3.4. Then $\varphi^{3}$ satisfies all the axioms in Theorem 3.1 except AN.

(4) For every spatial game $(N, v, p)$, let $N_{n d}(N, v, p)$ be the set of non-dummies, and let $\varphi^{4}$ assign 1 to player $i$ such that $p_{i}$ is lexicographically maximal among all $p_{j}, j \in N$ (so $p_{i}$ has maximal second coordinate among all points $p_{j}$ with maximal first coordinate); and 0 to all other players. Then $\varphi^{4}$ satisfies all the axioms in Theorem 3.1 except RI. Observe that $\varphi^{4}$ can be seen as resulting from the Dirac distribution that puts probability one on the issue $(1,0)$, using the second coordinate of positions for tie-breaking.

(5) Define

$$
\varphi_{i}^{5}(N, v, p)=\left\{\begin{array}{cl}
\frac{1}{\left|N_{n d}\right|} & \text { if } i \in N_{n d} \\
0 & \text { otherwise. }
\end{array}\right.
$$


Then $\varphi^{5}$ satisfies all the axioms in Theorem 3.1 except PI. To see the latter, suppose $N=\{1, \ldots, 4\}, p_{1}=(0,0), p_{2}=(1,1), p_{3}=(2,2), p_{4}=(0,2)$, and $p^{\prime}=p$ except that $p_{1}^{\prime}=(0,1)$. Then $\varphi_{4}^{5}\left(N, u_{N}, p\right)=\frac{1}{3}$ whereas $\varphi_{4}^{5}\left(N, u_{N}, p^{\prime}\right)=$ $\frac{1}{4}$.

Remark 3.14. We show that also the (implicit) nonnegativity requirement on a spatial power index cannot be dispensed with. Recall that this condition is (only) used in the proof of Lemma 3.11, which shows that the power of a player $i$ decreases if the angle at the extreme point $p_{i}$ of the polytope of the player positions increases. Without Lemma 3.11 we still have that $\varphi_{i}\left(N, u_{N}, p\right)=\frac{1}{2}(1-$ $q$ ) for a spatial game $\left(N, u_{N}, p\right)$ without dummies, if the angle at $p_{i}$ is equal to $q \pi$ for some $q=m / 2^{h}, h, m \in \mathbb{N}, m<2^{h}$. We will now define a power index which still satisfies this property and all axioms in Theorem 3.1, with sum of the powers of the players equal to one, but which violates nonnegativity. To this end, let $g$ : $\mathbb{R} \rightarrow \mathbb{R}$ be an additive function (i.e., $g(x+y)=g(x)+g(y)$ for all $x, y \in \mathbb{R}$ ) such that $g(x)=x$ for all $x \in \mathbb{Q}$ but not for all $x \in \mathbb{R} .^{5}$ We construct a spatial power index $\psi$ by defining it for all $\left(N, u_{N}, p\right), N \subseteq \mathbb{N}$, such that no player $i \in N$ is a dummy. By DP and EPC, $\psi$ is then extended to all spatial games. Let $\left(N, u_{N}, p\right)$ be a spatial game without dummies. Let the angle at $p_{i}, i \in N$, be $x_{i} \pi$. Then we define $\psi_{i}\left(N, u_{N}, p\right)=\frac{1}{2}\left(1-g\left(x_{i}\right)\right)$ for all $i \in N$. Since $\psi$ depends only on the angles in the polytope determined by the constellation $p$, it follows that $\psi$ satisfies AN, RI, and PI. Also, $\sum_{i \in N} \psi_{i}\left(N, u_{N}, p\right)=\frac{1}{2}\left(|N|-g\left(\sum_{i \in N} x_{i}\right)\right)=$ $\frac{1}{2}(|N|-(|N|-2))=1$, where the first equality follows from additivity of $g$. Since $g(x) \neq x$ for some $x \in \mathbb{R}$, it follows that $\psi \neq \Phi$. Consequently, nonnegativity must be violated.

Remark 3.15. The usual dummy condition would only require that dummy players obtain zero. We now show that this condition would not be sufficient to characterize the Owen-Shapley spatial power index. Define the power index $\psi$ as follows: for every spatial game $(N, v, p)$,

$$
\psi(N, v, p)=\left\{\begin{array}{cc}
\Phi(N, v, p) & \text { if }|N| \neq 3 \\
\varphi^{5}(N, v, p) & \text { if }|N|=3
\end{array}\right.
$$

where $\varphi^{5}$ was defined above: for $|N|=3, \varphi^{5}$ still satisfies PI. The spatial power index $\psi$ satisfies EPC, AN, RI, and PI, but not DP; however, it satisfies the weaker requirement that dummies obtain zero. Observe that, for $|N|=3, \psi$ is not continuous in the constellation $p$ : if none of the players is a dummy but one of the positions converges to a dummy position then the associated power remains at $\frac{1}{3}$ and thus does not converge to zero.

\section{Concluding remarks}

We characterized the Owen-Shapley spatial power index by means of five axioms. A limitation of our approach is that it is restricted to constellations and issues in

\footnotetext{
${ }^{5}$ The existence of such a function $g$ can be shown using the Axiom of Choice.
} 
$\mathbb{R}^{2}$. It is straightforward to extend the axioms to the case of constellations and issues in $\mathbb{R}^{m}$ for $m>2$, and it is safe to conjecture that the Owen-Shapley spatial power index satisfies these extended axioms. A proof of an eventual converse, however, seems to be more involved. Imposing continuity with respect to the constellation while weakening the dummy property DP to the requirement that dummies obtain zero might help here, cf. Remark 3.15.

As far as we are aware, this is a first axiomatic characterization of a spatial power index. Further research may concern characterizations of alternative power indexes, as proposed in the literature.

\section{References}

Alonso-Meijide JM, Fiestras-Janeiro MG, García-Jurado I (2011) A new power index for spatial games. In: Modern Mathematical Tools and Techniques in Capturing Complexity Understanding Complex Systems, pp. 275-285

Benati S, Marzetti GV (2013) Probabilistic spatial power indexes. Social Choice and Welfare 40:391-410

Derks J, Haller H (1999) Null players out? Linear values for games with variable support. International Game Theory Review 1:301-314

Dubey P (1975) On the uniqueness of the Shapley value. International Journal of Game Theory 4:131-139

Dubey P, Einy E, Haimanko O (2005) Compound voting and the Banzhaf index. Games and Economic Behavior 51:20-30

Einy E (1987) Semivalues of simple games. Mathematics of Operations Research 12:185-192

Einy E, Haimanko O (2011) Characterization of the Shapley-Shubik power index without the efficiency axiom. Games and Economic Behavior 73:615621

Grofman B, Owen G, Noviello N, Glazer A (1987) Stability and centrality of legislative choice in the spatial context. The American Political Science Review 81:539-553

Martin M, Nganmeni Z, Tchantcho B (2014) The Owen and Shapley spatial power indices: a comparison and a generalization. Working paper, THEMA, Cergy Pontoise

Owen G (1971) Political Games. Naval Research Logistics Quarterly 18:345354

Owen G (1995) Game Theory. Academic Press, San Diego 
Owen G, Shapley LS (1989) Optimal location of candidates in ideological space. International Journal of Game Theory 18:339-356.

Passarelli F, Bar J (2007) Preferences, the agenda setter, and the distribution of power in the EU. Social Choice and Welfare 28:41-60

Shapley LS (1977) A comparison of power indices and a non-symmetric generalization. Paper P5872, Rand Corporation, Santa Monica, CA

Shenoy PP (1982) The Banzhaf power index for political games. Mathematical Social Sciences 2:299-315

Straffin jr PD (1994) Power and stability in politics. Chapter 32 in: Handbook of Game Theory and Economics Applications, eds. Aumann RJ, Hart S. Elsevier, Amsterdam 